\title{
Industrialization Development of Traditional Arts and Crafts Intangible Cultural Heritage Under Mixed Reality Technology
}

\author{
Chenhui $\mathrm{Wu}$
}

\author{
Suan Sunandha Rajabhat University \\ 563216281@qq.com
}

\begin{abstract}
The mixed reality technology has developed rapidly in recent years, which has promoted the technological reform in all walks of life. The mixed reality technology has the interactive nature of real-time feedback between reality and virtual, and sets up an information loop of interactive feedback, which provides a new idea for the industrialization development of traditional arts and crafts intangible cultural heritage. This paper first analyzes the mixed reality technology, and then explores the concrete application of mixed reality technology in the industrialization development of traditional arts and crafts intangible cultural heritage, so as to realize the inheritance and development goal of traditional arts and crafts intangible cultural heritage.
\end{abstract}

Keywords: mixed reality technology; traditional craft; fine arts; intangible cultural heritage; industrialization

\section{INTRODUCTION}

Under the background of rapid development of information technology, mixed reality technology came into being and was widely used in various fields. The application of mixed reality technology in the industrialization development of traditional arts and crafts intangible cultural heritage can make the public communicate in the integration of virtual world and real world, and enhance the sense of cultural experience. At the same time, it can deepen the public's understanding of intangible cultural heritage from various angles, and then establish a correct concept of protection and inheritance of traditional arts and crafts intangible cultural heritage, so as to truly realize the industrialization development. Arts and crafts, as an outstanding representative of Chinese folk culture, has unique attributes and presents different cultural characteristics in different periods. In order to realize the industrialization development of traditional arts and crafts intangible cultural heritage, it is particularly necessary to apply mixed reality technology scientifically in the process of modernization to meet the needs of sustainable development[1].

\section{RESEARCH ON MIXED REALITY TECHNOLOGY}

\subsection{The Meaning of Mixed Reality Technology}

Mixed reality technology is a new visual digital environment created by the combination of virtual world and real world. Based on the rules and things of the real world, the mixed reality technology presents the information in the real scene in the virtual digital environment, creates a new visual environment and realizes the information interaction between the real world and the virtual world. The mixed reality technology mainly includes the sensing technology and the underlying processing and transmission technology[2]. The sensing technology transmits the human motion information to the corresponding processing equipment, which makes an immediate response to the human motion. At present, the common mixed reality technology uses head display and hand peripherals to capture, and uses cameras, infrared rays, lidar, gravity accelerometer and gyroscope for real-time sensing. In the mixed reality technology, the underlying processing and transmission technology is the most critical part of the operation. Virtual information and human body need a lot of calculations to respond in real time, and the movement and perception of human body 
can be generated into concrete figures. In addition to the above-mentioned technologies, mixed reality technology mainly relies on $3 \mathrm{D}$ to realize imaging, which can bring more real interactive experience to people, and then truly integrate into the virtual space environment[3].

\subsection{Characteristics of Mixed Reality Technology}

First, the interactivity of instant feedback. Because the sensing system of mixed reality technology can receive real-time information feedback in the actual experience process, including image, vibration, vision, sound and other feedback, the content scene can simulate many ideal states according to the corresponding real-time action pictures. In the medical field, mixed reality technology can be used in surgery simulation to improve the success rate of surgery. In the field of design, mixed reality technology can be applied in quantitative design. In the field of education, mixed reality technology can be used for interactive learning and simulation experiments. Second, the mutual transformation between reality and virtuality. In the field of virtual digital technology, mixed reality technology, virtual reality technology and augmented reality technology are the three most prominent technologies at present. Virtual reality technology can effectively simulate people's various perceptions in the natural environment; augmented reality technology is mainly based on the real environment, enhancing some features of the real environment; the mixed reality technology is based on the real environment, which is not a completely closed virtual world, but a virtual construction based on the real world, paying more attention to the transformation between the real world and the virtual world, and digitally reconstructing the real world. Thirdly, the digital transmission and storage hybrid reality technology is essentially a high-speed information processing technology, which can collect real-world information and store it digitally through the integrated processing of the processor. Digital decomposition and reconstruction of information is the main feature of mixed reality technology, which can be used to collect information and obtain digital images based on the real world[4].

\section{THE SIGNIFICANCE OF MIXED REALITY TECHNOLOGY APPLIED TO THE INDUSTRIALIZATION DEVELOPMENT OF TRADITIONAL ARTS AND CRAFTS INTANGIBLE CULTURAL HERITAGE}

\subsection{It is Conducive to Improving the Benefits of Traditional Arts and Crafts Intangible Cultural Heritage}

Intangible cultural heritage of traditional arts and crafts is the cultural content created by long-term production and life of working people. It has been inherited and developed for nearly a thousand years and occupies an important position in cultural construction[5]. Under the background of people's increasing demand for spiritual culture, the intangible cultural heritage of traditional arts and crafts is gradually developing towards industrialization, which can not only meet people's multicultural needs, but also promote the inheritance of intangible cultural heritage of traditional arts and crafts, thus obtaining good economic and social benefits.

\subsection{It is Conducive to Enhance the Public Visual and Auditory Experience}

The application of mixed reality technology in the industrialization development of traditional arts and crafts intangible cultural heritage can help the public to break through the perception in a limited range, deeply understand the connotation of intangible cultural heritage from multiple angles, and establish a good historical understanding value. At the same time, the mixed reality technology can make the distance between the public and the traditional arts and crafts intangible cultural heritage continuously narrow, make the public feel the expression of cultural diversity and draw nutrition from the intangible culture, so that the intangible cultural heritage of traditional arts and crafts can continue to be inherited and developed in modern society.

\subsection{It is Conducive to Promoting the Sustainable Development of Traditional Arts and Crafts Intangible Cultural Heritage}

The mixed reality technology records and displays the intangible cultural heritage of traditional arts and crafts in a different way from the traditional one, and will not be affected by the change of space or scene. The mixed reality technology presents the original appearance of traditional arts and crafts intangible cultural heritage to people from different angles through digital simulation, which can not only enhance the effectiveness of cultural communication, but also achieve the goal of cultural heritage protection and greatly promote the industrialization development of traditional arts and crafts intangible cultural heritage[6].

\section{THE FUNCTION OF MIXED REALITY TECHNOLOGY APPLIED TO THE INDUSTRIALIZATION DEVELOPMENT OF TRADITIONAL ARTS AND CRAFTS INTANGIBLE CULTURAL HERITAGE}

The intangible cultural heritage of traditional arts and crafts can realize all-round protection of intangible 
cultural heritage by using mixed reality technology. The involved images, videos, sounds, words and physical products are digitized and displayed to the public in a virtual way for people to browse, touch, learn and spread. As a high-tech technology, mixed reality technology can permanently store information collected by computers in the cloud, support real-time virtual calculus, and reproduce intangible cultural heritage of traditional arts and crafts in real scenes.

\subsection{Protection Function of Mixed Reality Technology}

Intangible cultural heritage can be roughly divided into two categories: visible but untouchable and visible and touchable. Intangible cultural heritage of traditional arts and crafts belongs to visible and touchable type. Mixed reality technology can record the specific process of production and display products vividly. In the past, the industrialization development of traditional arts and crafts intangible cultural heritage had limitations in product categories, while the mixed reality technology collected images, sounds and other information through digital technology and transformed it into permanent information storage, which fully protected intangible cultural heritage of traditional arts and crafts, and played an active role in the later remanufacturing and cultural research[7].

\subsection{Display and Dissemination Function of Mixed Reality Technology}

Mixed reality technology can not only store the information in the intangible cultural heritage of traditional arts and crafts, but also carry out real interaction and all-round display through virtual space, so that people are immersed in the cultural situation. At the same time, the mixed reality technology breaks through the limitation of time and space of the traditional arts and crafts intangible cultural heritage in the past, which can make people have access to more information, make the intangible cultural heritage of traditional arts and crafts enter the public's field of vision and awaken the public's cultural identity, thus promoting the protection and development of the traditional arts and crafts intangible cultural heritage. In addition, the technology of mixed reality can cross the barrier of time and space. In the past, people could only learn about the intangible cultural heritage of traditional arts and crafts by watching relevant materials, while people can go back to different historical periods and watch the intangible cultural heritage of traditional arts and crafts beyond the limitations of time and space, which is more conducive to spreading cultural elements.

\subsection{The Function of Using Mixed Reality Technology to Create a Business Platform}

At present, the intangible cultural heritage of traditional arts and crafts is facing the problem of insufficient successors. With the change of social and economic model, the inheritance of intangible cultural heritage of family style, workshop style and mentoring style cannot continue. In particular, the intangible cultural heritage of traditional arts and crafts has extremely high technological requirements, and the successors of arts and crafts need to study hard, and few people can persevere. Arts and crafts are different from craft replicas on the assembly line, and there is often a problem that it is difficult to reproduce[8]. Works contain the activation of traditional arts and crafts and the inheritance of history and culture. However, mixed reality technology can make traditional arts and crafts digitally reappear, promote intangible cultural heritage products to become mass consumer goods, create a good business platform, and promote virtual arts and crafts in global commercial circulation.

\section{PROBLEMS OF MIXED REALITY TECHNOLOGY APPLIED TO THE INDUSTRIALIZATION DEVELOPMENT OF TRADITIONAL ARTS AND CRAFTS INTANGIBLE CULTURAL HERITAGE}

The mixed reality technology used in the industrialization development of traditional arts and crafts intangible cultural heritage can transform the invisible material of human beings in the real world into perceptible information, enhance the visual effect of the public, and build a cultural exchange platform, thus promoting the innovation of non-genetic inheritance mode of traditional arts and crafts. However, there are some problems in the application of mixed reality technology, which deserve attention and analysis. First, although mixed reality technology has increased the economic value of traditional arts and crafts intangible cultural heritage, if it is not used properly, it may lead to the entertainment of cultural communication, which violates the original intention of technology application. Secondly, with the development of information technology, the speed of cultural communication is accelerating, which has brought impact to the exhibition of traditional arts and crafts intangible cultural heritage. Although the application of mixed reality technology can achieve the expected display effect of traditional arts and crafts intangible cultural heritage, there are some limitations in the application of technology, which requires the support of high-level equipment and cannot achieve the same coverage as mobile terminals. Finally, the software platform of mixed reality technology is still in the primary stage of development and research. At present, there are few companies and teams engaged in 
the field of mixed reality in the world, and the operation of related industries is insufficient in terms of manpower and technology. These are all the problems of mixed reality technology in the industrialization development of traditional arts and crafts intangible cultural heritage, and it is necessary for relevant personnel to formulate optimization measures according to specific conditions in order to give full play to the role of mixed reality technology[9].

\section{SUGGESTIONS ON THE APPLICATION OF MIXED REALITY TECHNOLOGY IN THE INDUSTRIALIZATION DEVELOPMENT OF TRADITIONAL ARTS AND CRAFTS INTANGIBLE CULTURAL HERITAGE}

\subsection{Strengthen the Collection of Intangible Cultural Heritage Resources of Traditional Arts and Crafts by Mixed Reality Technology}

Collection is the premise of the application of mixed reality technology in the intangible cultural heritage of traditional arts and crafts. Relevant information should be collected and produced by camera equipment to lay the foundation for virtual display. Collection work requires relevant personnel to track information collection on the basis of not destroying the intangible cultural heritage of traditional arts and crafts, not only requiring collectors to have a high professional level, but also knowing the types and characteristics of traditional arts and crafts intangible cultural heritage in detail, so as to improve the value of cultural research and display the elegance of traditional arts and crafts intangible cultural heritage from different angles. Specifically, the intangible cultural heritage resources of traditional arts and crafts can be transformed and utilized by using mixed reality technology, so as to create digital intangible cultural heritage exhibition halls of traditional arts and crafts, realize cultural innovation, artistic innovation and scientific innovation, raise public's cultural awareness, and encourage the public to actively participate in cultural protection.

\subsection{With the Help of Mixed Reality Technology to Deepen the Experience of Traditional Arts and Crafts Intangible Cultural Heritage}

It is one of the main purposes of industrialization to help mankind know the history of traditional arts and crafts intangible cultural heritage, and the cohesion of a nation needs the support of cultural identity, so the social education function of traditional arts and crafts intangible cultural heritage should be enhanced. As for the traditional arts and crafts intangible cultural heritage, it mainly includes cultural knowledge, traditional crafts, national spirit and other elements. In the industrialization construction of traditional arts and crafts intangible cultural heritage, mixed reality technology should be integrated to meet the diversified experience of the public, and the origin, distribution and system of traditional arts and crafts intangible cultural heritage should be introduced through virtual reality, so that intangible cultural heritage has a three-dimensional sense and visitors can feel the feeling of crossing history. At the same time, the audio-visual experience unit is established, and with the help of hardware and software such as display platform, imaging equipment, control equipment, etc., the visual and auditory effects of dynamic, virtual and realistic combination can be realized, so that visitors can be present at the scene, increase the interaction of visitors, and explore more forms of performances, so that people can be integrated into the cultural atmosphere and further expand the influence of traditional arts and crafts intangible cultural heritage[10].

\section{CONCLUSION}

To sum up, the application of mixed reality technology in the industrialization development of traditional arts and crafts intangible cultural heritage is innovative cultural communication, but the application of mixed reality technology needs to follow the principle of scientificity and moderation to avoid the entertainment problem of cultural communication. At present, the mixed reality technology is still immature, and human-computer interaction needs a lot of high-tech support. Blind application to the industrialization development of traditional arts and crafts intangible cultural heritage is prone to various problems, which can not really highlight the cultural value. Therefore, we should pay attention to the research and development of mixed reality technology, and reasonably apply it to the collection, protection and dissemination of traditional arts and crafts intangible cultural heritage, so as to realize the industrialization development goal of intangible cultural heritage of traditional arts and crafts.

\section{ACKNOWLEDGMENTS}

I deeply appreciate Asst. Prof. Dr. Akapons Inkuer of Suan Sunandha Rajabhat University for his guidance and encouragement to this paper.

\section{REFERENCES}

[1] Guo Tingting, $\mathrm{Fu}$ Yao.Thoughts on Industrialization Development of Traditional Arts and Crafts Intangible Cultural Heritage under Mixed Reality Technology[J].Journal Of Hubei Open Vocational College,2019,32(21):96-97.

[2] Li Fang.Research on Digital Development of Intangible Cultural Heritage Based on Mixed 
Reality Technology[J].Science And Technology \& Innovation,2016(12):40-40.

[3] Fu Yao.Application of Mixed Reality Technology in the Exhibition of Intangible Cultural Heritage[J].Journal of Jiamusi Vocational Institute,2019(6):201-202,204.

[4] Fu Yao.Research on the Application of Mixed Reality Technology in the Inheritance of Intangible Cultural Heritage Education in Colleges and Universities[J].China Journal Of Multimedia \& Network Teaching,2019(7Z):222-224.

[5] Li Tingting.Communication of Intangible Cultural Heritage in Public Space Based on AR-VR Hybrid Technology[J].Electronics and Software Engineering,2019(12):144.

[6] Xie Jia.Research on Innovation of Communication Path of Intangible Cultural Heritage[J].Journalism Lover,2021(10):61-63.

[7] Zhuang Wenjie, Tong Mingwen.Communication Strategy of Intangible Cultural Heritage Video Resources from the Perspective of Intelligence[J].Journal of Huazhong Normal University(Humanities and Social Sciences),2021,60(4):93-106.

[8] Wei Huanyun.Protection and Inheritance of Intangible Cultural Heritage[J].Packaging World,2021(5):73.

[9] Ma Zhiyao, Gao Shan.Research on Intangible Cultural Heritage and Cultural Identity[J].Qilu Realm of Arts,2021(4):109-114.

[10] Huo Yanchang, Zhao Qiaoyan.Activation and Inheritance of Intangible Cultural Heritage - Based on the Investigation of Lu Silk Weaving Skills in Shanxi[J]. Journal of The Beifang University Of Nationalities(Philosophy and Social Sciences Edition),2021(1):46-52 